\section{Instrumentos de participación pública en plusvalías urbanas en una ciudad turística intermedia: el caso de Bariloche, Argentina}

\author{
Mariana Dondo ${ }^{1}$ \\ ORCID: https://orcid.org/0000-0001-7921-8579 \\ Correo electrónico:mdbuhler@unrn.edu.ar
}

Héctor Martín Civitaresi ${ }^{1}$

ORCID: https://orcid.org/ 0000-0001-6375-1678

\author{
Laura Valeo ${ }^{1,2}$ \\ ORCID: https://orcid.org/0000-0001-7120-7541
}

\section{María Alejandra Nussbaum 1,2 \\ ORCID: https://orcid.org/0000-0002-4764-9670}

\section{Resumen}

En la actualidad, las ciudades intermedias turísticas presentan un crecimiento poblacional acelerado, fragmentación social y espacial y una especulación excesiva que distorsiona el valor del suelo local. Asimismo, sus gobiernos locales dependen económicamente de transferencias de recursos de otras jurisdicciones, lo que supone un debilitamiento de su autonomía relativa ante otros niveles de decisión política y un condicionamiento para cumplir con su rol de dinamizador del desarrollo sostenible. El objetivo del artículo es indagar sobre los instrumentos de participación pública en las plusvalías urbanas, desde un punto de vista conceptual y empírico. Analizamos dos casos de estudio en la ciudad de Bariloche, Argentina: la obtención de tierras para la nueva terminal de ómnibus y la obra del puente del Ñireco. Los resultados indican que, si están bien diseñados en sus aspectos técnicos y tienen legitimidad, los instrumentos contribuyen a obtener recursos para financiar el desarrollo urbano y aportan a una estructura de financiamiento de los gobiernos locales más equitativa. Por un lado, el gobierno local obtiene recursos, no necesariamente monetarios, sobre los cuales puede decidir libremente, otorgándole una mayor autonomía en la planificación urbana. Por ejemplo, creando valor en el suelo de propiedad privada y apropiándose de una parte para destinarlo a proyectos de interés público. Por otro lado, al disminuir el beneficio esperado de especular con la tierra, se contribuye a regular de manera positiva el mercado del suelo. Aportamos evidencia empírica útil para que otros gobiernos locales apliquen estos mecanismos y promuevan un desarrollo de las ciudades más equitativo.

Palabras clave

Bariloche, financiamiento del desarrollo, gestión del suelo, plusvalía urbana

\section{Instruments of public participation in urban capital gains in an intermediate tourist city: The case of Bariloche, Argentine}

\author{
Filiación institucional: \\ 1. Universidad Nacional de Río Negro, CIETES, Bariloche, Argentina. \\ 2. Municipalidad de Bariloche, Argentina.
}

Financiamiento: El artículo fue desarrollado en el marco del proyecto de investigación financiado por la Universidad Nacional de Río Negro "Estructura, funcionalidades y trayectoria histórica del gobierno local en Bariloche. Un estudio con énfasis en su grado de autonomía y su impacto sobre el desarrollo socioeconómico local". Una versión previa fue presentada en el II Congreso Internacional de Desarrollo Territorial (2020).

\begin{abstract}
Currently, intermediate tourist cities have accelerated population growth, social and spatial fragmentation, and excessive speculation that distorts the value of local land. Likewise, their local governments depend economically on resource transfers from other jurisdictions, which implies a weakening of their relative autonomy from other levels of political decision-making and conditioning to fulfill their role as a catalyst for sustainable development. The aim of the article is to investigate the instruments of public participation in urban capital gains, from a conceptual and empirical point of view. We analyze two case studies in the city of Bariloche, Argentina: obtaining land for the new Bus Terminal and the construction of the Nireco Bridge. The results indicate that, if they are well designed in their technical aspects and have legitimacy, the instruments contribute to obtaining resources to finance urban development and contribute to a more equitable financing structure for local governments. On the one hand, the local government obtains resources, not necessarily monetary, on which it can decide freely, granting it greater autonomy in urban planning. For example, creating value in privately owned land and appropriating part of it to be used for projects of public interest. On the other hand, by reducing the expected benefit of speculating with land, they regulate the land market positively. We provide useful empirical evidence for other local governments to apply these mechanisms and promote more equitable development of cities.
\end{abstract}

\section{Keywords}

Bariloche, development financing, land management, urban capital gain 


\section{Introducción}

La reproducción de las ciudades en Argentina ha resultado de la interacción de tres lógicas de funcionamiento: por un lado, la del Estado que, a nivel local, habitualmente no cuenta con recursos para construir la ciudad. Por otro lado, la del mercado, en la cual actores inmobiliarios tienen una lógica de maximizar las ganancias y apropiarse de las rentas del suelo generadas por el esfuerzo colectivo y, como contrapartida, la necesidad de sectores que buscan reproducir su vida y acceder al derecho a la vivienda y al hábitat (Reese, 2014). Cada uno de estos actores tiene distintos intereses, grados de poder de negociación y de acción en la construcción de la ciudad. Como resultado, nuestras ciudades presentan características que se sintetizan en segregación urbana; convivencia de una alta densidad de población en zonas sin servicios y una baja densidad en zonas servidas; ciudades extendidas, caras y con alto consumo de recursos naturales; falta de obras, servicios básicos y de utilidad pública; ocupación irregular del suelo; privatización del espacio público; una alta especulación relativa al suelo que encarece el acceso a la vivienda y también afecta la competitividad de las actividades productivas, entre otras.

En este contexto, las municipalidades deben cumplir un rol relevante. Por un lado, porque se estima que el $90 \%$ de la población es urbana y vive en ciudades gobernadas localmente por municipalidades (Hagedorn et al., 2015). Por otro, porque estas han tenido que asumir nuevos desafíos vinculados con políticas de desarrollo, a partir de procesos de descentralización y transferencias de competencias desde el nivel central a los niveles subnacionales en las últimas décadas. A los servicios urbanos tradicionalmente provistos por el gobierno local, se le sumaron las demandas de aquellos descentralizados, así como de aquellos que no son provistos de manera suficiente por otros niveles (por ejemplo, promoción del empleo, provisión de suelo urbano, ordenamiento territorial y cuidado ambiental) (López Accotto et al., 2012). Sin embargo, en la práctica, las municipalidades frecuentemente se ven limitadas para ejercer el rol que crecientemente se les demanda, condicionadas por sus capacidades institucionales reales y por la escasa disponibilidad de recursos sobre los cuales pueden decidir plenamente su asignación (Cravacuore, 2016; Flores et al., 2018; Gorenstein et al., 2010; López Accotto y Macchioli, 2015). Esta problemática se reitera para cualquier política pública local, incluida aquella de interés en este artículo, a saber, la implementación de recursos para desarrollo urbano.

El objetivo de este artículo es indagar acerca de los instrumentos de participación pública en las plusvalías urbanas para la promoción del desarrollo de ciudades intermedias turísticas. Estos instrumentos permiten que el Estado recupere parte de la valorización capitalizada en la propiedad inmueble generada por su intervención, sea mediante obra pública o por actos administrativos (cambios en normas y regulaciones) (Jaramillo, 2009; Smolka, 2013). De este modo, se moviliza para el beneficio de la sociedad, parte o la totalidad del aumento del valor del suelo generado por acciones ajenas al propietario, logrando que, quienes más se benefician de la dinámica del mercado de suelo, sean también quienes más contribuyan a cubrir el costo de la urbanización.

Algunas preguntas que guían la investigación son las siguientes: ¿contribuyen estos instrumentos a superar las limitaciones de financiamiento del desarrollo urbano de los gobiernos locales?, ¿aportan a una estructura de financiamiento municipal más progresiva y orientada a la promoción de la competitividad local?, ¿bajo qué condiciones ayudan a alcanzar objetivos vinculados con la garantía de derechos (el suelo cumpliendo con su función social) y de equidad (que el Estado pueda redistribuir parte de las rentas generadas por el esfuerzo de la comunidad)?

Se ha considerado el caso de ciudades turísticas intermedias ya que, generalmente, reciben excedentes económicos locales y foráneos traducidos en inversiones inmobiliarias, esperando una alta rentabilidad de la mano de la actividad turística. Esto genera cambios en el uso del suelo, la especulación y valorización inmobiliaria favorecen el desarrollo de grupos económicos concentrados que condicionan la aplicación de políticas urbanas que permitan afrontar la fragmentación del espacio urbano, 
el incremento de asentamientos informales y la exclusión social (De Mattos, 2008; Lolich, \& Guevara, 2015).

El artículo se organiza de la siguiente manera: en primer lugar, se presentan los instrumentos de participación en plusvalías desde un punto de vista conceptual, analizando distintos argumentos que justifican su implementación. También se expone una sistematización, no exhaustiva, de antecedentes sobre diseño y aplicación de estos instrumentos, poniendo foco en los utilizados de manera más frecuente en las ciudades latinoamericanas. A continuación, se caracteriza la ciudad de Bariloche como caso de estudio. En la sección siguiente se discuten dos casos de aplicación de instrumentos alternativos, adaptados a la normativa y al contexto local. Por un lado, la aplicación de la normativa de renta diferencial urbana para la obtención de las tierras necesarias para la construcción de una nueva terminal de ómnibus en la ciudad. Por otro lado, la posible aplicación de la recuperación de plusvalías en el caso de la obra de un puente de acceso a la ciudad sobre el río Ñireco. El artículo se cierra con una sección de reflexiones finales acerca del potencial de estos instrumentos para la obtención de recursos para promover el desarrollo equitativo de las ciudades.

\section{Aspectos conceptuales: instrumentos de participación pública en plusvalías urbanas}

Estos instrumentos buscan captar la renta de la tierra generada sin la realización de un esfuerzo productivo por parte del propietario. El aumento en el valor de la tierra puede ser ocasionado, por un lado, por la realización de obras de infraestructura (pública o privada), de hecho, existe evidencia respecto de que esta inversión genera una riqueza superior al valor invertido, y que este incremento depende de la localización (Peinado et al., 2018; Soria Caballero, 2019). Por otro lado, acciones administrativas y cambios normativos que modifican la categoría de uso del suelo (urbana/rural) y la zonificación (industrial/residencial/comercial) habilitan un mayor aprovechamiento (factores de ocupación/alturas máximas) y lo valorizan. La plusvalía originada en intervenciones públicas puede ser apropiada por el gobierno local a través de diferentes mecanismos que significan una fuente de recursos, monetarios o no, y contribuyen a desincentivar la especulación.

Diversos argumentos se pueden esbozar para justificar la implementación de mecanismos de captación de plusvalías. En primer lugar, se plantea que el derecho de propiedad debe ser limitado cuando se contrapone al interés general, y que implica obligaciones en beneficio de la sociedad. En este sentido, la participación y distribución de plusvalías es un asunto de equidad ciudadana, ya que se apoya en el principio de función social de la propiedad y en el enriquecimiento sin causa justa de los propietarios (Garzón, 2019). Por ejemplo, la propiedad privada debe respetar ciertas normas para no contraponerse al derecho de acceder a una vivienda, en los casos en que la especulación o la tenencia de vivienda ociosa elevan el precio en el mercado.

En segundo lugar, estos instrumentos contribuyen a la regulación del mercado del suelo, en particular, donde hay una alta especulación como generalmente ocurre en las ciudades turísticas. En el mercado de suelo, los propietarios se comportan como monopolistas, ya que cada parcela es única e irrepetible en sus características, en especial, su localización. El precio del suelo deriva del beneficio que se espera obtener por los productos inmobiliarios que se van a construir sobre él (Jaramillo, 2009). La máxima ganancia que puede obtenerse del suelo depende del acceso, los equipamientos, servicios, localización, aprovechamiento permitido, etc., factores que, en gran parte, no están vinculados con el esfuerzo del propietario, sino con el planeamiento urbano. El cambio en las normas urbanas puede aumentar el precio del suelo y eso es un estímulo a la especulación inmobiliaria, ya que los propietarios, solamente por esperar que se realicen determinadas intervenciones, se enriquecen. El gobierno puede intervenir para desincentivar esa especulación o limitar el enriquecimiento sin causa justa, apropiándose de ese plusvalor generado.

Finalmente, las municipalidades necesitan disponer de más recursos para promover el desarrollo urbano. 
Estos instrumentos proveen recursos aportados en mayor proporción por contribuyentes con más riqueza, por lo que contribuyen con equidad a la estructura de recaudación. Además, pueden mejorar la competitividad económica, ya que influyen en la estructura de costos (a través de los precios de alquileres, por ejemplo) y desincentivan las actividades rentistas/especulativas que inhiben la inversión productiva.

Si bien muchas municipalidades están facultadas legalmente para implementar instrumentos de captación de plusvalías, su implementación aún es poco habitual en algunas ciudades latinoamericanas. Para que estos instrumentos puedan ser aplicados y alcanzar sus objetivos, se deben considerar las siguientes cuestiones:

- Los instrumentos tienen que ser legales y legítimos y tiene que haber voluntad política de implementarlos. Esto significa que, como condición necesaria, tienen que estar habilitados en el marco normativo vigente. Un paso en ese sentido es avanzar en las autonomías efectivas de los municipios, para que puedan gestionar su propio presupuesto y diseñar sus instrumentos tributarios y no tributarios de recaudación.

Sin embargo, la legalidad no garantiza la legitimidad necesaria para que la movilización de las plusvalías realmente ocurra, sobre todo en contextos donde la ciudadanía tiene la percepción de que ya existe una alta presión impositiva o una alta restricción al derecho de propiedad. Para ello, se ha indicado que la disposición a pagar es mayor cuando los aportantes tienen claridad acerca del destino de los recursos o cuando las propiedades valorizadas tienen un valor de cambio, más que un valor de uso (Cáceres y Sabatini, 2001). Esto es, si la propiedad valorizada fue construida para vender, esa venta ocurre en el corto plazo y genera el flujo de ingresos que permite afrontar el pago. Mientras que, en el caso de las propiedades que tienen un uso residencial, esa mayor valorización se traducirá en dinero recién en el momento de la venta del inmueble, que puede ocurrir en un largo plazo. Esto es, la disposición a pagar es mayor (y posible) en el momento en que la plusvalía se transforma en liquidez y no en el que es generada. La captación de plusvalías implica que contribuyentes con mayor riqueza aporten recursos que actualmente no están aportando y, por lo tanto, se espera que estas medidas sean resistidas (López Accotto et al., 2018). Sin embargo, los gobiernos locales que tienen voluntad política de implementarla pueden valerse de su poder de negociación frente a los desarrolladores urbanos y grupos de poder económico, ya que tienen la potestad sobre la normativa que regula el uso del suelo $\mathrm{y}$, por tanto, de sus decisiones depende que se puedan llevar a cabo los proyectos (Hagedorn et al., 2015).

- Cuestiones técnicas y operativas: por un lado, el contenido y la redacción de la norma deben ser claros para quienes la pueden aplicar. Por otro lado, el procedimiento de aplicación debe ser simple y poco costoso. Finalmente, deben existir las capacidades técnicas para medir la valorización, identificar las propiedades afectadas, contar con catastros o sistemas de información adecuados. Sin embargo, existen antecedentes que demuestran que, aun sin tener la posibilidad de valuar de manera precisa, los instrumentos pueden ser diseñados e implementados con impactos positivos (Duarte y Baer, 2013).

- Para que la implementación de estos instrumentos sea redistributiva y aporte a la disminución de las desigualdades socioespaciales, es necesario que la inversión de los recursos recuperados se localice en una zona diferente a la que generó la plusvalía o, si se invierten en la misma zona valorizada, que impliquen beneficios para los sectores más pobres, como transporte público o vivienda de interés social. Caso contrario, se entra en un ciclo regresivo de valorización, recuperación, reinversión y nueva valorización de la zona, que es a la vez generadora y beneficiaria de plusvalía. La inversión debe estar orientada a cerrar la brecha de acceso a la urbanización, proveyendo vivienda a sectores medios y bajos que no satisfacen su demanda en el mercado o generando valorización en zonas de menor desarrollo relativo, mediante el aporte de infraestructura, obra pública, etc. 
A continuación, se describen algunos de los instrumentos más frecuentemente utilizados en América Latina para captar plusvalías. Es interesante destacar que estos instrumentos pueden tomar innumerables formas, tanto en su diseño como en su aplicación (hechos generadores de plusvalías que se consideran, porcentaje de la valorización que movilizan, forma y momento de pago, destino de los recursos, entre otros), dando lugar a la posibilidad de adaptarse a los contextos locales. Incluso, se observa que instrumentos similares tienen denominación diferente en los distintos países. Ordenamos los instrumentos presentados en tres grupos que se describen a continuación:

\section{Aquellos que permiten captar plusvalía generada por} las fluctuaciones propias de las dinámicas urbanas

En su aplicación, la desventaja puede ser la gentrificación (implica la movilización de sectores que son propietarios históricos y que no pueden afrontar el aporte).

\section{Contribución por desarrollo o valorización.}

Este instrumento amplía la contribución por mejoras para captar plusvalías y no solamente recuperar costos de la inversión pública. Se incluyen como hechos imponibles la inversión y otras intervenciones públicas como cambios de normativa que incrementan los precios del suelo. Un ejemplo es la contribución diseñada en el municipio de Trenque Lauquen en Argentina donde, aún sin poder medir de manera precisa la valorización de la tierra, el municipio logró captar plusvalías (Duarte y Baer, 2013). En particular, en el caso de la aprobación de loteos, una empresa pública municipal se encargó de diseñar la subdivisión y realizar las obras de infraestructura, financiadas por el propietario y pagadas con dinero o lotes. Se determinó que el $12 \%$ de los lotes del nuevo fraccionamiento sería cedido al municipio a modo de pago por las plusvalías generadas. De esta manera, el municipio le resuelve el fraccionamiento al propietario, quien puede lotear sin invertir dinero, y también se apropia de lotes para aumentar la oferta de suelo urbanizado. En Isunza-Vizuet et al. (2021), Páramo Lopera y López Morales (2020), y Furtado y Acosta (2020) se presentan casos de aplicación de la contribución por valorización en Uruguay, México, varias ciudades de Colombia (Cali, Bogotá, Medellín, Manizales, entre otras) y Brasil.

Impuesto a la propiedad inmueble.

Se cobra sobre la tenencia de la propiedad inmueble sobre la base de su valor fiscal. En su diseño habitual, no es un instrumento de captación de plusvalías, ya que aun si las bases imponibles se actualizan continuamente, logra recuperar un porcentaje muy bajo del plusvalor (el impuesto grava la plusvalía, pero es débil recuperándola para el Estado) ${ }^{1}$. Sin embargo, se puede adaptar el instrumento para movilizar plusvalías. Por un lado, si la base del impuesto fuera solo el valor de la tierra (sin mejoras), o si se gravara de manera separada la tierra de las mejoras, se captaría la valorización no relacionada con esfuerzos del propietario (Soria Caballero, 2019).

1 Los impuestos gravan el plusvalor. En contraposición, los instrumentos en cuestión recuperan la plusvalía generada, esto es, la renta que tiene un origen social, generada sin esfuerzo del propietario. El impuesto inmobiliario (predial) es débil captando plusvalías. El siguiente ejemplo clarifica esta diferencia:

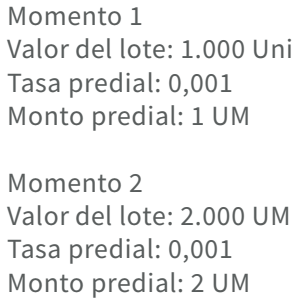

Es decir que, si bien el valor del lote y el impuesto se incrementaron en un 100\%, solo se logró capturar el 0,1\% de la valorización (1 UM adicional/1.000 de valorización). Un instrumento de captación del 30\% de la plusvalía permitiría capturar 300 UM en este caso. 
En Perló Cohen y Zamorano Ruiz (2001) se presentan experiencias en Mexicali y Tijuana, México, sobre la implementación del impuesto basado en el suelo, lo que resultó en importantes incrementos de la recaudación y una más efectiva captura de plusvalías. En América Latina la base suele ser sobre el valor fiscal del inmueble completo (tierra y mejoras), sin embargo, es frecuente que se cobren tasas más elevadas sobre la tierra ociosa. Por ejemplo, en Niterói (Río de Janeiro, Brasil), el impuesto territorial para inmuebles no edificados es tres veces mayor al impuesto para inmuebles con edificación (Furtado y Acosta, 2020). En otros casos, se aplican tasas crecientes en el tiempo a los inmuebles ociosos para incentivar el máximo aprovechamiento de la tierra (Furtado y Acosta, 2020). Por otro lado, se puede aplicar un incremento temporario de la tasa a los beneficiarios de determinada intervención pública. Un ejemplo es la sobretasa cobrada a los residentes de la ciudad de Buenos Aires en el caso de la obra de extensión del subterráneo, más un adicional cobrado a los propietarios de inmuebles localizados hasta 400 metros de distancia de la estación nueva. Otro ejemplo es el aporte voluntario del $10 \%$ adicional del impuesto predial cobrado en Bogotá para financiar inversión social (Furtado y Acosta, 2020).

\section{Aquellos que permiten captar plusvalía sobre una ciudad planificada}

\section{Zonificación condicionada.}

Los propietarios pueden pedir cambio de uso o parámetros urbanos y la municipalidad los concede a cambio de una contraprestación equivalente a la valorización.

Potencialidades del suelo, bonos por densidad, concesión onerosa del derecho de construcción.

La municipalidad planifica su política urbana, establece un coeficiente básico de aprovechamiento para la ciudad e indica en qué áreas podrían hacerse ampliaciones en los parámetros de densidad o área cubierta. La municipalidad vende los derechos de construcción que exceden el básico, que pueden ser pagados en efectivo, en infraestructura pública o vivienda social. Un ejemplo de aplicación es el Régimen Especial por Mayor Aprovechamiento por Incremento de Altura de Rosario, Argentina, que permite obtener un mayor aprovechamiento constructivo mediante el pago de una contribución compensatoria (Peinado et al., 2018). Otro ejemplo lo constituyen las operaciones interligadas aplicadas en Brasil, por medio de las cuales el Estado, aplicando cambios normativos, permite mayor densidad constructiva valorizando ciertas propiedades privadas. Este "suelo creado" es otorgado a los propietarios a cambio de recursos. Este instrumento se aplicó en el caso del Shopping West Plaza en Sao Paulo, Brasil, en el cual el gobierno otorgó un derecho de construcción tres veces mayor al original, a cambio del valor equivalente a la construcción de 810 viviendas de interés social (Sandroni, 2001).

Techo legal de densidad.

Este instrumento permite aprobar proyectos que excedan los parámetros establecidos, en caso de que el planeamiento urbano lo apruebe, a cambio de que propietario pague al municipio el valor equivalente a los terrenos necesarios para construir el excedente aprobado.

Otros instrumentos y políticas urbanísticas con los cuales suelen combinarse los instrumentos de captación de plusvalías

\section{Convenios urbanísticos.}

Son los instrumentos jurídicos que formalizan acuerdos entre el municipio y actores privados, mixtos o públicos, para la realización de acciones de urbanización que generan beneficios mutuos. Involucran permisos y excedentes en algunos parámetros, a cambio de la movilización de recursos a favor del bien público, como cesiones de tierra, equipamiento urbano, ejecución o financiamiento de obras de servicios, vialidades y espacios públicos, y construcción de vivienda social. Según Hagedorn et al. (2015), el modelo de distribución de los valores generados por la acción pública puede seguir el principio de los tres tercios: 1) 1/3 del plusvalor pertenece al desarrollador del proyecto que garantiza la rentabilidad; 2) 1/3 a la municipalidad, que asegura las funciones públicas a través de la inversión de lo recuperado; 3) 1/3 es la masa negociable, en función de los costos que cada uno asume, la calidad exigida a las construcciones, entre otros argumentos. En general, 
este mecanismo se utiliza en el caso de aprobación de grandes proyectos urbanos, como son: Puerto Madero (Buenos Aires, Argentina), la regeneración del frente costero en Rosario (Argentina) (Peinado et al., 2018; Pons Giner, 2015) o la transformación del área industrial del eje Tamanduatehy, en Santo André, Brasil, hacia otros usos económicos (Furtado y Acosta, 2020).

\section{Fondos Municipales de Desarrollo Urbano.}

Consisten en una cuenta de capital que se provee de aportes provenientes de la captación de plusvalías y se destinan a financiar inversiones para el desarrollo urbano local.

\section{Bancos Municipales de Tierra.}

Posibilitan al municipio disponer de terrenos para la implementación de estrategias de desarrollo urbano, económico y social (Hagedorn et al., 2015). Los gobiernos locales adquieren tierra, en general cuando la coyuntura de precios es favorable, para vender en épocas de precios altos, concesionar, hacer loteos para la construcción de vivienda accesible y social, para la localización de equipamiento público, espacio público, prevenir especulaciones, entre otros.

\section{Empresas Municipales de Desarrollo Urbano.}

Participan como desarrolladoras en el mercado local y reinvierten las plusvalías generadas por su actividad para mejorar la calidad urbana y/o en proyectos urbanos con fines sociales.

\section{Caso de estudio: la ciudad de Bariloche, Argentina}

La ciudad de Bariloche tiene una población cercana a los 110 mil habitantes de acuerdo con el censo de 2010 (Instituto Nacional de Estadística y Censos [INDEC], 2010). Demográficamente es la ciudad más importante de la provincia de Río Negro y la segunda de toda la Patagonia Norte. Está alejada del centro político administrativo de la provincia (Viedma, la capital, se encuentra a más de 900 km; mientras que Buenos Aires está ubicada a más de $1.600 \mathrm{~km})$. Bariloche se ha convertido en una ciudad con un importante grado de centralidad en la región (economías de localización, ofertas educativas, alojamiento de niveles de administración de gobierno provincial o nacional, entre otras) $\mathrm{y}$, atendiendo a su funcionalidad regional, se la podría identificar como una ciudad intermedia (Civitaresi y Colino, 2019).

La estructura productiva de Bariloche se encuentra sobre la base de la industria turística. La ciudad, uno de los centros turísticos más importantes del país y reconocida a nivel mundial, recibe alrededor de 700 mil visitantes al año con una marcada estacionalidad en invierno y verano (Kozulj, 2018; Monasterio, 2006). Junto con la oferta de atractivos naturales, se encuentran una variedad de complejos productivos relacionados, llevados adelante en su mayoría por empresas de tipo familiar (chocolates artesanales, alimentos gourmet, ahumados, derivados de frutas finas, hongos, cervezas artesanales, entre otros) (Civitaresi et al., 2017; Colino y Savarese, 2019). Además, la ciudad tiene como particularidad una alta concentración del complejo científico tecnológico del país. Se destaca el clúster tecnológico Bariloche, un conglomerado de 22 empresas e instituciones que conforman un sector tecnológico competitivo a nivel nacional e internacional (Lugones y Lugones, 2004). Sin embargo, la escasa diversificación productiva de Bariloche hace que se constituya en una economía marcada por la estacionalidad y las oscilaciones de la actividad turística vinculadas con variables poco controlables localmente, como el manejo del tipo de cambio, la política de transporte, tarifas de gas o valor del combustible (Malvicino et al., 2018). Esto va en detrimento de emprendimientos productivos locales alternativos, capaces de impulsar un desarrollo local económica y socialmente más equilibrado (Oglietti y Colino, 2011).

Estas características de ciudad intermedia turística han determinado una expansión acelerada del ejido urbano avanzando sobre áreas rurales, con baja densidad, escasa planificación y desigualdades socioterritoriales. La ciudad se ha ido completando de este a oeste sobre la costa del lago Nahuel Huapi con el asentamiento de actividades vinculadas principalmente con el turismo, viviendas de clase media y media-alta y segundas residencias. 
Paralelamente, la ciudad se ha ido expandiendo hacia el sur, la pampa de Huenuleo, de geografía compleja, con suelos inundables y de menor valor económico e infraestructura urbana, donde tienden a localizarse los sectores populares más vulnerables de la comunidad (Matossian, 2014, 2015; Medina, 2017). Este fenómeno no es exclusivo de Bariloche; la relación entre mercantilización del ocio y especulación inmobiliaria ha sido estudiada para diferentes destinos turísticos (Clifford, 2003; De Sousa et al., 2016; González y Mantecón, 2014).

En cuanto a las finanzas locales, la municipalidad presenta una débil autonomía económica y un presupuesto con bajo margen de maniobra para financiar sus objetivos de política (Dondo et al., 2019). En el año 2019, solo el $54 \%$ de sus ingresos totales fueron propios, mientras que el resto fueron transferidos desde la provincia y la nación. Del total de ingresos de libre disponibilidad (propios o transferidos desde otras jurisdicciones), el $88 \%$ se destinó a cubrir gastos corrientes, quedando un escaso remanente para financiar políticas. La estructura de financiamiento propio se compone de múltiples instrumentos tributarios, pero, así como en la mayor parte de los municipios argentinos, una alta proporción de la recaudación recae en la Tasa por Inspección Seguridad e Higiene que tiene un impacto regresivo y debilita la competitividad local (Dondo et al., 2019). Si analizamos la ejecución presupuestaria de 2019 desde la perspectiva del gasto, observamos que el $3 \%$ de los gastos fueron destinados a financiar obra pública.

El marco jurídico vigente en la ciudad habilita la implementación de instrumentos de captación de plusvalía. En primer lugar, la Constitución Nacional Argentina incorpora la función social del suelo a través de la inclusión del Pacto de San José de Costa Rica, donde se determina que "toda persona tiene derecho al uso y goce de sus bienes. La ley puede subordinar tal uso y goce al interés social" (artículo 21). De este modo, se separa el derecho de propiedad del derecho de construir. El otorgamiento del derecho a construir es una competencia de la administración pública y, en Argentina, corresponde a los gobiernos municipales. En segundo lugar, la Constitución de la Provincia de Río Negro se plantea en el preámbulo "promover la función social de la propiedad" y "fortalecer la autonomía municipal”. Además, establece la facultad de los municipios de crear y recaudar impuestos siempre que no vuelvan a gravar hechos ya gravados. Esto da lugar a que los municipios utilicen otras fuentes de recursos además de las tasas y las transferencias de niveles superiores de gobierno. En tercer lugar, la Carta Orgánica de la Municipalidad de Bariloche declara la función social de la propiedad y, a través del diseño de planes urbanos y el establecimiento de indicadores urbanos, regula el derecho de construcción de los propietarios del suelo.

En particular, la Ordenanza 2733-CM-16 aprueba y establece la aplicación del derecho a participar de la renta diferencial urbana, señalando que este es un mecanismo que:

apunta a corregir uno de los fenómenos más inequitativos de nuestra ciudad: el funcionamiento espontáneo y sin regulación alguna del mercado de la tierra que, con el aporte de importantes recursos fiscales, genera incrementos considerables en los precios de ciertos terrenos. Esta situación deriva en grandes beneficios hacia un puñado de propietarios que mantienen en esto un papel pasivo, mientras los fondos para soportar el desarrollo de la ciudad resultan crónicamente escasos. Mediante el cobro de los derechos regulados en la presente Ordenanza se intenta brindar al Estado la capacidad de recuperar para la comunidad, al menos, una porción de los incrementos en los valores inmobiliarios que se encuentren íntimamente ligados a decisiones estatales, rescatando de esta manera para el conjunto de los ciudadanos parte de los frutos del esfuerzo colectivo. (Ordenanza 2733-CM-16).

Se aplica a todas las personas físicas o jurídicas propietarias o poseedoras de inmuebles ubicados en el ejido municipal. Los hechos generadores son el establecimiento o la modificación de parámetros urbanísticos (factor de ocupación del suelo, factor de ocupación total, área de edificación y superficie máxima); cambios de uso de parcela solicitados por el interesado, modificación de 
área de implantación y la autorización de subdivisión del suelo cuando medie el cambio de uso o parámetros. El índice de participación municipal es del $12 \%$ para el caso de modificación de usos del suelo y del $20 \%$ en el resto de los casos. El pago puede ser en dinero, pero también en inmuebles, obra de infraestructura de servicios públicos, áreas de recreación o equipamiento. Las únicas excepciones son las obras públicas (de cualquier nivel del Estado) y los proyectos declarados de interés social por parte del Concejo. A su vez, se crea un Fondo Municipal de Desarrollo Urbano en el cual se depositarán los pagos por renta diferencial urbana.

Sintetizando, observamos que Bariloche presenta múltiples problemas urbanos y carencias de equipamiento e infraestructura, a la vez que cuenta con un escaso margen de recursos disponibles para financiar el desarrollo. En la ciudad, al menos dos fuentes de ingresos corrientes tienen como destino específico el financiamiento de obras de infraestructura y equipamiento urbano. Por un lado, los ingresos de capital asociados al recupero de obras y captación de plusvalías que, en 2019, significaron apenas un 0,3\% de los ingresos propios (\$5 millones). Por otro lado, la Ecotasa, que retribuye por los servicios turísticos y la inversión en infraestructura turística que realiza la municipalidad (conservación patrimonial, mejoramiento de sitios, accesos al lago, miradores, servicios de información, entre otros), pagada por todos turistas mayores a 14 años que se hospeden en la ciudad. Su recaudación alcanzó en 2019 \$69 millones (5,3 \% de recursos propios corrientes). Entendemos que la implementación de instrumentos de captación de plusvalías es una oportunidad para movilizar una mayor cantidad de recursos necesarios para financiar la brecha de desarrollo urbano y, a la vez, regular el mercado de suelo.

\section{Implementación de instrumentos en Bariloche}

Desde su aprobación, en el año 2016, la ordenanza se ha aplicado en algunos casos, a través de la realización de convenios urbanísticos. Por ejemplo, por el cambio de volumetría y ocupación del suelo para la ampliación de un hotel; para la ampliación de un supermercado, ocupando el tercio libre en planta baja y alta; y en el cambio de uso del suelo para la realización de un proyecto urbano por parte de una desarrolladora inmobiliaria, en el cual la renta diferencial se abonó en el equivalente en obras de intubación de un arroyo y la construcción del área de transferencia de transporte público y estacionamiento.

En esta sección, desarrollamos dos casos de aplicación de la ordenanza (Figura 1):

- un caso en el que la municipalidad logró acceder, a través de la renta diferencial urbana, a las tierras necesarias para la ubicación de la nueva terminal de ómnibus de Bariloche (Ordenanza 2871/17);

- un caso potencial de aplicación de la movilización de plusvalías, en el cual actualmente solo se cobran contribuciones por mejoras para financiar parcialmente el costo de la obra.

\section{Aplicación de la Ordenanza de Renta Diferencial urbana en la gestión de tierras para establecer la terminal de ómnibus}

Desde hace años se identifica la necesidad de ampliar y reubicar la actual terminal de ómnibus de la ciudad. Conforme a ello, en el año 2017, el gobierno de la provincia de Río Negro negoció la construcción de una nueva terminal con la empresa Entretenimientos Patagónico SRL, que opera dos salas de casino en Bariloche, como contraprestación por la renovación de la concesión por 15 años. La empresa haría la inversión por 4,4 millones de dólares en un plazo no mayor a 18 meses y el municipio debía aportar la tierra para su emplazamiento.

La Secretaría de Desarrollo Urbano realizó un análisis multicriterio para seleccionar la mejor localización de la terminal y, en función de ello, propuso a la empresa Las Victorias SRL, propietaria de un terreno en el área seleccionada, la modificación de la traza de un fraccionamiento previamente autorizado y la implementación de nuevos parámetros para el sector. Ambos hechos generarían una renta diferencial urbana, que sería captada por la municipalidad por medio de 
Figura 1

Ubicación de los dos casos de estudio en la ciudad de Bariloche

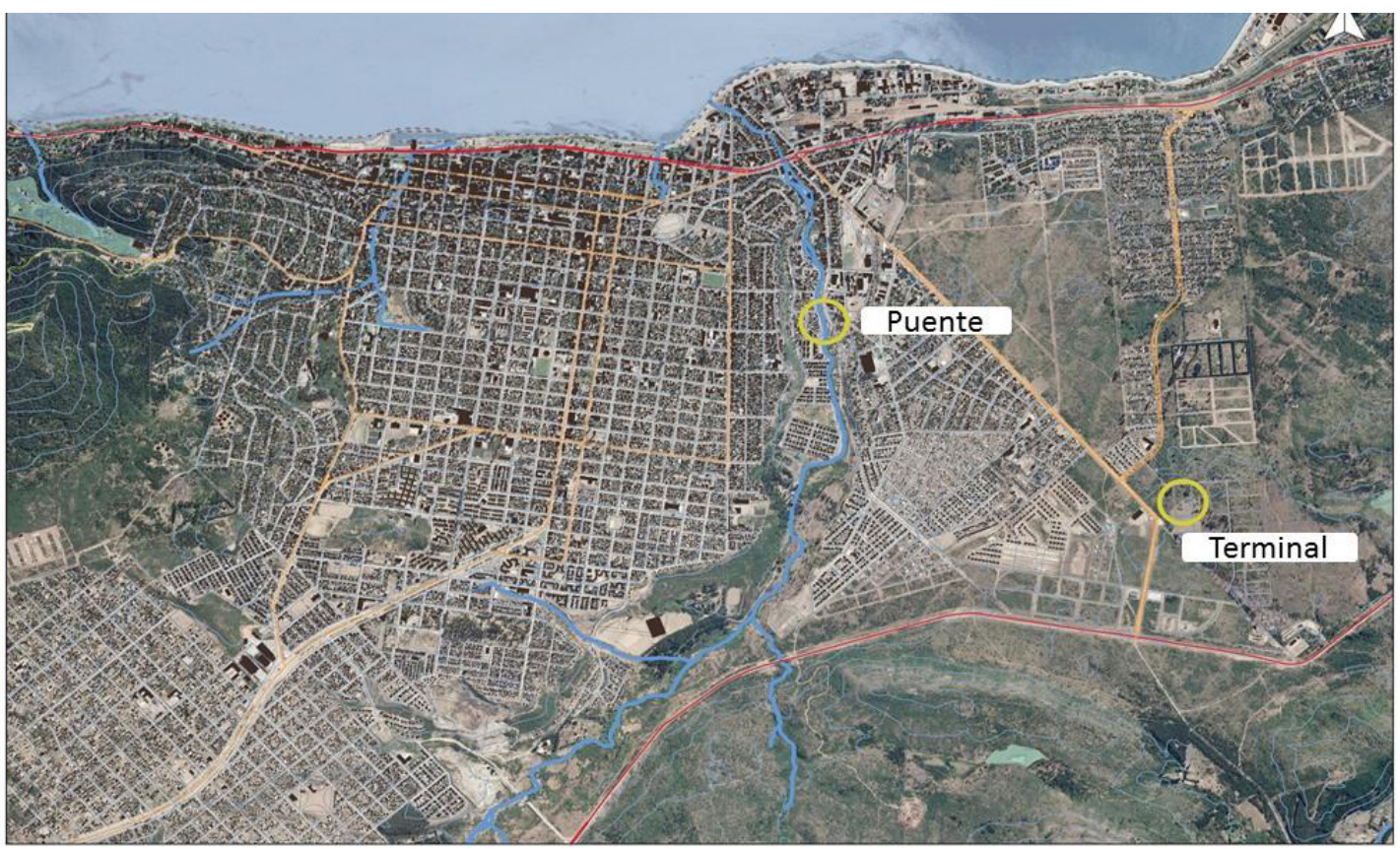

Figura 2

El nuevo puente sobre el arroyo Nireco de acceso a la ciudad

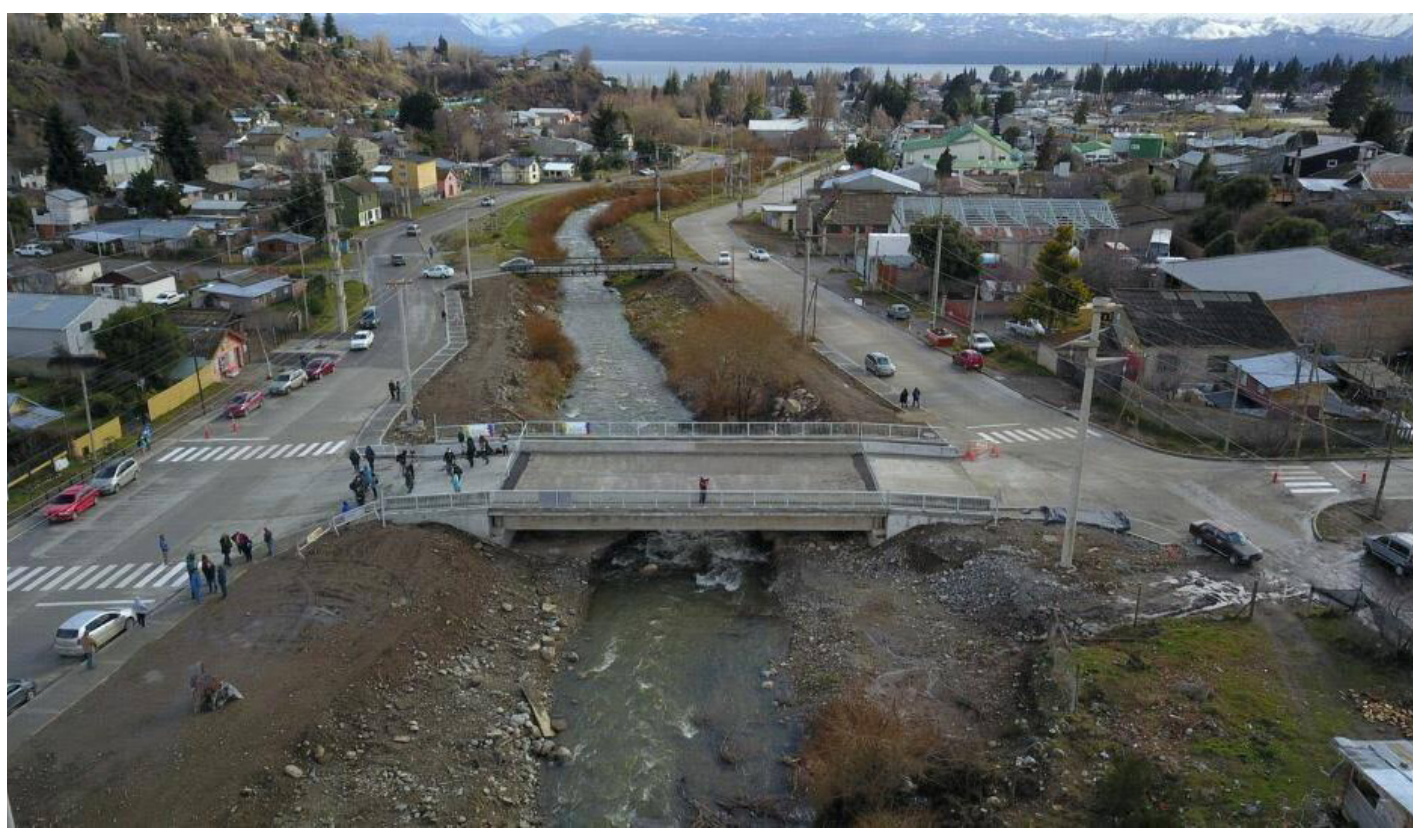

Nota: Adaptado de Se inauguró el nuevo puente del arroyo Ñireco (27 agosto de 2020) [Fotografía]. Semanario Bariloche Hoy. Día a día. https://semanariobariloche.com.ar/actualidad/se-inauguro-el-nuevo-puente-del-arroyo-nireco/ 
Tabla 1

Cálculo de la renta diferencial urbana

\begin{tabular}{lll}
\hline & Hecho generador de RDU1: modificación fraccionamiento \\
\hline Superficie de parcelas comercializable $\left(\mathrm{m}^{2}\right)$ & 81.359 & $(1)$ \\
\hline Tasa RDU & $12 \%$ & $(2)$ \\
\hline Superficie a ceder al municipio $\left(\mathrm{m}^{2}\right)$ & 9.763 & $(3)=(1){ }^{*}(2)$ \\
\hline & Hecho generador de RDU2: modificación parámetros urbanísticos \\
\hline m2 construibles- & 263.015 & $(4)$ \\
\hline Parámetros originales & 284.844 & $(5)$ \\
\hline Parámetros modificados & 21.829 & $(6)=(5)-(4)$ \\
\hline Diferencia m ${ }^{2}$ construibles & $20 \%$ & $(7)$ \\
\hline Tasa RDU & 12.230 & $(8)$ \\
\hline Valor $m^{2}$ construcción & 53.393 .587 & $(9)=(6) *(7) *(8)$ \\
\hline RDU en $\$$ & 704 & $(10)$ \\
\hline Valor del $m^{2}$ en la zona $(\$)$ & 75.843 & $(11)=(9) /(10)$ \\
\hline Superficie a ceder al municipio $\left(\mathrm{m}^{2}\right)$ & 85.606 & $(12)=(3)+(11)$ \\
\hline Superficie total $\left(\mathrm{m}^{2}\right)$ & &
\end{tabular}

la cesión de tierra necesaria para la construcción de la terminal. Además, la empresa cedería otros lotes adicionales, que permitirían al municipio completar vacíos urbanos en el área, uno de los objetivos de la política urbana primordial para la mejora de la conectividad, la integración social y la eficiencia en el uso de los recursos en cuanto a infraestructura y equipamiento (Ordenanza 2871/17).

Entre el municipio y la empresa se firmó un convenio urbanístico. En la Tabla 1 se presentan los cálculos realizados aplicando la normativa vigente para la obtención de los $\mathrm{m}^{2}$ que la empresa debía ceder a la municipalidad. Por un lado, la empresa debía ceder el $12 \%$ de la superficie comercializable que surge de la modificación de la autorización de subdivisión (9,7 mil $\mathrm{m}^{2}$ ). En segundo lugar, debía ceder el $20 \%$ del valor que representan los $\mathrm{m}^{2}$ construibles que se adicionan por los cambios en los parámetros urbanísticos. Dado que la municipalidad en este caso necesitaba recibir tierra, y no efectivo, ese valor se convirtió nuevamente a $\mathrm{m}^{2}$ con base en una tasación del valor comercial promedio del $\mathrm{m}^{2} \mathrm{de}$ parcela con servicios básicos en la zona. De este modo, por la modificación de parámetros la empresa tenía que ceder al municipio $75 \mathrm{mil} \mathrm{m}^{2}$, totalizando $85,6 \mathrm{mil} \mathrm{m}^{2}$.

En este caso, los instrumentos de participación en plusvalías urbanas fueron utilizados satisfactoriamente para movilizar los recursos necesarios para lograr el equipamiento urbano y cumplir con los objetivos de la política urbana. Sin embargo, la terminal no fue construida, porque la empresa Entretenimientos Patagónicos SRL incumplió el contrato, argumentando que la devaluación y la inflación ocurridas desde el momento de la firma de este dificultaron la inversión.

\section{Aplicación de una sobretasa a la Tasa de Servicios Municipales a beneficiarios directos de obra del Puente del Ñireco}

Actualmente, la ordenanza que regula la participación de la municipalidad en las plusvalías urbanas no considera como hecho generador de las mismas la realización de obra pública. El instrumento que se implementa en estos casos la Contribución por Mejoras (Ordenanza 2973-CM-18) que, tal como está diseñado actualmente, busca recuperar una parte o la totalidad del costo de una obra pública con la contribución de aquellos propietarios de inmuebles que aumentan de valor por su ejecución. Previo al llamado a licitación de la obra, hay una instancia en la que los propietarios beneficiarios pueden oponerse a la realización o su forma de pago. En el caso de que menos del $30 \%$ de los propietarios se oponga, entonces la obra es realizada y el pago de la contribución, obligatorio. El monto recaudado se destina al Fondo de Desarrollo Urbano. 
Tabla 2

Recaudación adicional por sobre tasa para captación de plusvalía

\begin{tabular}{|c|c|c|c|c|c|c|}
\hline \multirow{2}{*}{ Barrio } & \multicolumn{2}{|c|}{ Recaudación Tasa Servicios Municipales } & \multirow{2}{*}{ Contribuyentes } & \multirow{2}{*}{$\%$ Rec. } & \multirow{2}{*}{$\%$ Contr. } & \multirow{2}{*}{$\begin{array}{c}10 \% \text { adicional en } \\
\text { USD }\end{array}$} \\
\hline & Pesos & USD & & & & \\
\hline Ñireco & 700.193 & 42.282 & 111 & $0,6 \%$ & $0,2 \%$ & 4.228 \\
\hline 112 viviendas & 58.173 & 3.513 & 111 & $0,0 \%$ & $0,2 \%$ & 5.817 \\
\hline Lera & 1.601 .943 & 96.736 & 927 & $1,3 \%$ & $1,7 \%$ & 9.674 \\
\hline San Francisco & 1.384 .318 & 83.594 & 670 & $1,1 \%$ & $1,2 \%$ & 8.359 \\
\hline Bariloche & 125.090 .833 & 7.553 .794 & 54.443 & & & 28.078 \\
\hline
\end{tabular}

Nota: Tabla elaborada sobre la base de la recaudación municipal, 2017.

Este instrumento podría ser reformulado, de modo de que no solamente permita recuperar el costo de una obra que el municipio financia a los vecinos, sino también apropiarse parte de las plusvalías generadas. Si bien no existe actualmente la información para estimar de manera precisa el incremento del valor inmobiliario debido a la ejecución de una obra, se podría comenzar aplicando una sobretasa temporaria a la tasa de servicios municipales, a pagar por aquellos beneficiarios directos de la obra.

Un caso posible de aplicación en la ciudad es la obra de construcción de un puente sobre el arroyo Ñireco (Figura 2), que mejora las condiciones de acceso y la conectividad entre el este y la ciudad. Para realizarla, la municipalidad tomó préstamos del Plan Castello provincial y prevé recuperar el costo de la obra aplicando la Contribución por Mejoras. Debido a que el puente es uno de los accesos principales a la ciudad, se considera que los beneficiarios de la obra son todos los residentes. Por eso, se plantea que la contribución sea pagada por todos los contribuyentes de la Tasa de Servicios Municipales. Esta obra, además de mejorar el acceso a la ciudad, incrementará el valor de los inmuebles de la zona de influencia.

En la Tabla 2 se presentan los resultados de un ejercicio simple en el cual se estima el monto aproximado que podría recuperarse en concepto de plusvalías, aplicando una sobretasa del $10 \%$ sobre la tasa de servicios municipales a los inmuebles de los barrios más directamente beneficiados. Como el último dato disponible de la recaudación desagregada por barrio es del año 2017, los montos se dolarizaron.

En este caso, observamos que la recaudación adicional sería de aproximadamente 28 mil dólares, que se destinarían al Fondo de Desarrollo Urbano. Si bien el monto es insuficiente para financiar proyectos urbanos de envergadura, sí podría contribuir a financiar programas de mejoramiento de vivienda o de infraestructura comunitaria.

\section{Conclusiones}

En este trabajo presentamos una discusión conceptual sobre los instrumentos de captación de plusvalías, que permiten a los gobiernos municipales avanzar en el cumplimiento de distintos objetivos. Por un lado, obtener recursos, no necesariamente monetarios, sobre los cuales decidir libremente y que le viabilizan hacer política de desarrollo urbano. De este modo, contribuyen a alcanzar una mayor autonomía de los gobiernos subnacionales. Por otro lado, estos instrumentos disminuyen el beneficio esperado de especular con la tierra, regulando de manera positiva el mercado del suelo. La participación de la municipalidad en la plusvalía generada puede ser lograda a través de múltiples instrumentos y las normativas frecuentemente habilitan la innovación en su diseño.

En el trabajo avanzamos también en el análisis de aplicación de dos mecanismos diferentes de movilización de plusvalías en la ciudad de Bariloche, donde la normativa habilita estos instrumentos y ya existen algunos antecedentes de aplicación. El análisis de los resultados nos permite aportar evidencia a las preguntas de investigación planteadas.

En primer lugar, nos habíamos preguntado si los instrumentos contribuyen a obtener recursos para financiar el desarrollo urbano y que, además, aporten a una estructura de financiamiento de los gobiernos locales más equitativa y que promueva la competitividad. Los resultados muestran que la recuperación de las plusvalías generadas otorga recursos importantes a los gobiernos locales. Si bien la recaudación adicional estimada en el segundo caso fue insuficiente para invertir en proyectos 
grandes, esos recursos monetarios sí pueden aportar a un fondo que permita financiar mejoramientos. El caso de la terminal muestra que los instrumentos son eficaces en resolver el acceso a tierra necesaria para el equipamiento urbano y la vivienda social. En este caso, además, probablemente la legitimidad (y la cobrabilidad asociada) del instrumento sea mayor, ya que es explícito para el propietario cómo el gobierno, mediante el cambio de normas, valoriza el suelo. En el caso del puente, probablemente, la sobretasa sea percibida únicamente como una mayor carga de impuestos, sin detectar tan fácilmente cómo el Estado valorizó la propiedad privada mediante su inversión.

Respecto del aporte de estos recursos a la estructura de financiamiento municipal, muchas veces recostada sobre tributos regresivos, podemos observar que la recaudación se realiza sobre una base patrimonial, lo cual implica un avance en términos de equidad. Además, los instrumentos de captación de plusvalía pueden desestimular la especulación basada en el suelo, que tiene impactos negativos: hace que la oferta de suelo sea más rígida al dejarlo inmovilizado, muchas veces incluso servido, y por eso empuja los precios al alza. Tener altos precios de suelo disminuye la competitividad de las actividades productivas, ya que implica un alto costo fijo. Estos instrumentos, entonces, pueden potenciar la actividad productiva en detrimento de la rentística/especulativa.

En segundo lugar, nos habíamos preguntado en qué condiciones la participación pública en las plusvalías urbanas aporta para alcanzar objetivos vinculados con la garantía de derechos y de equidad. Como mencionamos anteriormente, la legalidad de los instrumentos es condición necesaria y, en caso de que la normativa no los habilite, probablemente el proceso deliberante se encuentre con la oposición de actores con poder económico y político que dificulten su reglamentación. Aquí se pone de manifiesto la importancia de contar con gobiernos locales que tengan poder de negociación y una gran voluntad política de avanzar en este sentido. La legitimidad, que garantiza que la participación pública sea efectivamente realizada, también tiene que ser lograda. A su vez, algunos aspectos técnicos deben considerarse para que su implementación sea posible. Sin embargo, como observamos en la comparación de los casos, aun sin la posibilidad de realizar estimaciones precisas de la valorización de las propiedades afectadas, la aplicación de estos instrumentos puede contribuir de manera importante a mejorar el acceso a la tierra para construir vivienda o infraestructura urbana. En este sentido, la normativa debería estar planteada de tal manera que habilite a innovar en el diseño y la forma de aplicación de los mecanismos para crear y recuperar plusvalor. $Y$ los funcionarios a cargo de implementarlos tendrán que contar con la información y preparación para hacerlo.

Respecto del poder redistributivo de los instrumentos, en los casos de aplicación se pueden identificar tres momentos: 1) Generación de plusvalía; 2) Participación (distribución de plusvalías entre el Estado y el propietario); y 3) Redistribución. En los momentos de participación y de redistribución se pueden analizar aspectos vinculados con la equidad (Garzón, 2019). En la participación, porque ahí es cuando se determina qué proporción de la plusvalía generada apropia el sector privado y cuánto el Estado, representando a la comunidad. En el caso de la terminal, el porcentaje fue el planteado en la norma (12\% y $20 \%$ según la fuente de valorización). En el caso del puente, no se estimó de manera precisa la valorización de las propiedades, ya que no se contaba con la información para hacer el ejercicio, por lo cual no fue posible saber de manera precisa la distribución entre Estado y propietario. En el momento de la redistribución también se pueden analizar aspectos vinculados con la equidad, porque ahí se determina si lo apropiado por el Estado es reinvertido en la zona valorizada previamente o es asignado en función del interés común, en particular, en el de los sectores más vulnerables o en otros sectores. En el caso de la terminal, los recursos se asignaron a la inversión en equipamiento urbano que favorece a gran parte de la población local, tanto por la vía de las mejores condiciones para el turismo como para el transporte de pasajeros. En el análisis de impacto sobre la equidad no solo es relevante a qué sector o con qué objetivos se asignan los recursos apropiados por el gobierno, sino también su magnitud (Iñíguez, 2012, 2014). En el caso del puente, podemos apreciar que los recursos son insuficientes 
para financiar proyectos urbanos de envergadura, sin embargo, si los fondos recaudados fueran invertidos en mejoramiento del barrio o las viviendas precarias (de la zona valorizada o de otras zonas de la ciudad), se podría ver un impacto redistributivo de la recuperación de plusvalías.

En el caso de Bariloche, podemos concluir que estos ejemplos de aplicación aportan evidencia en favor de la creación y participación pública en las plusvalías urbanas como mecanismo para promover el desarrollo. Estos instrumentos se encuentran al alcance de muchos gobiernos locales y pueden contribuir de manera importante a que el desarrollo de las ciudades sea más equitativo. ${ }^{45}$

\section{Referencias bibliográficas}

Cáceres, G. y Sabatini, F. (2001). Santiago de Chile: la recuperación de plusvalías puesta en perspectiva histórica. Recuperación de plusvalías en América Latina. Alternativas para el desarrollo urbano. Eurelibros.

Carta Orgánica de la Municipalidad de Bariloche. Art. 14. 15 de abril de 2007 (Argentina).

Civitaresi, H. M. y Colino, E. (2019). Turismo, transformaciones territoriales y resiliencia: Bariloche como evidencia de una ciudad turística intermedia argentina. REDER, Revista de Estudios Latinoamericanos sobre Reducción del Riesgo de Desastres, 3(1), 41-52. http://www.revistareder.com/ojs/ index.php/reder/article/view/22

Civitaresi, H. M., Niembro, A. A. y Dondo Bühler, M. B. (2017). Desafíos para desarrollar una agroindustria local. Hacia una tipología de productores de cerveza artesanal en Bariloche. Revista Pymes, Innovación y Desarrollo, 5(1), 41-62. https://www.researchgate.net/publication/338015354_ Desafios_para_desarrollar_una_agroindustria_local_Hacia una_tipologia_de_productores_de_cerveza_artesanal_en_ Bariloche
Clifford, H. (2003). Downhill Slide: Why the Corporate Ski Industry is Bad for Skiing, Ski Towns, and the Environment. Sierra Club Books

Colino, E. D. V. y Savarese, M. (29-30 de abril, 2019). Chocolates de Bariloche, la trama socio-productiva de un producto con identidad territorial [Sesión de conferencia]. II PreCongreso Argentino de Desarrollo Territorial y I Jornadas Patagónicas de Intercambio Disciplinar sobre Desarrollo y Territorio, Bariloche, Argentina.

Constitución de la Nación Argentina. Art. 21. 22 de agosto de 1994 (Argentina).

Constitución de la Provincia de Río Negro. Preámbulo. 3 de junio de 1988 (Argentina).

Cravacuore, D. (2016). Gobiernos locales en Argentina. En J. M. Ruano y C. Vial (Eds.), Manual de gobiernos locales en Iberoamérica (pp. 15-40). CLAD; Universidad Autónoma de Chile.

De Mattos, C. (2008). Globalización, negocios inmobiliarios y mercantilización del desarrollo urbano. En M. Córdova Montúfar (Ed.), Lo urbano en su complejidad: una lectura desde América Latina (pp. 35-62). FLACSO.

De Sousa, P.G., Matheus, E., \& Fragoso, S. (2016). From residential tourism to tourist real estate complexes: The appropriation of the coastalzone in the Northeast of Brasil by Tourist Real Estate activities. Ambiente $y$ Sociedade, 19(3), 177-198. https://doi.org/10.1590/18094422asoc141673v1932016

Dondo, M., Civitaresi, H.M. y Dursi Saadi I. (2019). Sustentabilidad financiera de los gobiernos subnacionales: un análisis exploratorio de la municipalidad de Bariloche. Revista Digital Desarrollo y Territorio, (6), 50-54. https://rid.unrn. edu.ar/handle/20.500.12049/4714 
Duarte, J. y Baer, L. (2013). Recuperación de plusvalías a través de la contribución por mejoras en Trenque Lauquen, provincia de Buenos Aires-Argentina. Lincoln Institute of Land Policy.

Flores, H. D., Gil, M., Altavilla, C. D. e Iparraguirre, E. (2018). Las decisiones del gasto público y el rol de los municipios en el desarrollo local en Argentina un abordaje desde la autonomía municipal y los actores sociales y políticos. TERRA: Revista de DesarrolloLocal,(4),1-31.https://doi.org/10.7203/terra.4.10894

Furtado, F. y Acosta, C. (2020). Recuperación de plusvalías urbanas en Brasil, Colombia y otros países de América Latina: conceptos, instrumentos e implementación (Documento de trabajo). Lincoln Institute of Land Policy. https://www.lincolninst.edu/sites/default/files/ pubfiles/2230_1564_Furtado_WP13FF1SP.pdf

Garzón, V. (2019). La participación del Estado en la plusvalía como una deuda ciudadana. Revista Ratio Juris, 14(28), 287-320. https://doi.org/10.24142/raju.v14n28a10

González, R. y Mantecón, A. (2014). Turismo y negocio inmobiliario: la crisis de un modelo de desarrollo. Tres estudios de casos de Canadá, Argentina y España. Estudios y Perspectivas en Turismo, 23(4), 685-705. http:/ rua.ua.es/dspace/handle/10045/40964

Gorenstein, S., Geymonat, A., Landriscini, G., Hernández, J., Preiss, O., Domeett, G., Donadoni, M., Napal, M., Urriza, G., \& Pacciaroni, C. (28-30 de octubre de 2010). Una revisión y algunas preguntas sobre ciudades intermedias. Explorando funciones en ciudades pampeanas y norpatagónicas [Sesión de conferencia]. XI Seminario Internacional RII de Investigadores sobre Territorio y Globalización. Universidad Nacional de Cuyo, Mendoza, Argentina.

Hagedorn, T., Panizoni, L., Borgna, B. y Magno, J. (2015). Guía de instrumentos urbanísticos. Secretaría de Asuntos Municipales. Ministerio del Interiory Transporte de Argentina.

Instituto Nacional de Estadística y Censos. (2010). Censo Nacional de Población, Hogares y Viviendas 2010. https:// www.indec.gob.ar/indec/web/Nivel4-Tema-2-41-135
Iñiguez, A. (2012). Tributos al modelo. Récords del presente y desafíos del futuro. Revista Entrelíneas de la Política Económica, (32), 3-80. http://sedici.unlp.edu. ar/handle/10915/18042

Iñiguez, A. (2014). Cómo enriquecer el sistema tributario argentino. Entrelíneas de la Política Económica, (7), 22-39. http:// sedici.unlp.edu.ar/bitstream/handle/10915/43429/Documento_ completo_.pdf?sequence $=1$ \&isAllowed=y

Isunza-Vizuet, G., Castro, E. y Munévar, C. (2021). La plusvalía como sistema definanciación urbana: estudio comparativo en Ciudad de México y Manizales, Colombia. Revista EURE (Santiago), 47(142), 229-248. https://doi.org/10.7764/eure.47.142.11

Jaramillo, S. (2009). Hacia una teoría de la renta del suelo urbano (2da edición). Ediciones Uniandes.

Kozulj, R. (2018). Plan Estratégico de Turismo de Bariloche (Informe preliminar). Mimeo.

Lolich, L., \& Guevara, T. (6-9 de julio de 2015). Sustainable developement in a touristic city with protected areas. A diagnostic study applied to an urb an area near the Nahuel Huapi National Park in Patagonia [Sesión de conferencia].5th Annual International Conferenceon Architecture,Atenas, Grecia.

López Accotto, A. y Macchioli, M. (Coord.) (2015). La estructura de la recaudación municipal en la Argentina: alcances, limitaciones y desafíos. Editorial Universidad Nacional de General Sarmiento.

López Accotto, A., Martínez, C., Grinberg, I. y Adaro, C. (2012). Nuevos modelos de gestión local: la articulación de políticas públicas y el protagonismo ciudadano a través de la implementación del presupuesto participativo en la Argentina. Revista Pueblos y Fronteras Digital, 7(13), 92-126. https://doi.org/10.22201/cimsur.18704115e.2012.13.111

López Accotto, A., Martínez, C., Mangas, M. y Paparas, R. (2018). Los impuestos a la riqueza en Argentina en una perspectiva comparada. Revista Economía y Desafíos del Desarrollo, 1(4). http://www.unsam.edu.ar/escuelas/ economia/revistaedd/3n2/ 
Lugones, G. y Lugones, M. (2004). Bariloche y su grupo de empresas intensivas en conocimiento: realidades $y$ perspectivas (Documento de trabajo $N^{\circ} 17$ ). Redes Centro de Estudios sobre Ciencia, Desarrolloy Educación Superior.

Malvicino, F. E., Civitaresi, H. M. y Dondo Bühler, M. B. (1920 de septiembre de 2018). Desarrollo regional, tipo de cambio y competitividad local. El impacto de las políticas macroeconómicas en la actividad turística de San Carlos de Bariloche [Sesión de conferencia]. XII Jornadas Nacionales de Economías Regionales: Estrategias y dialécticas territoriales ante la reconfiguración neoliberal. Bernal, Buenos Aires, Argentina.

Matossian, B. (2014). Inserción urbana y desigualdades sociales de migrantes recientes en San Carlos de Bariloche. Párrafos Geográficos, 13(2), 47-75. https://ri.conicet.gov. ar/handle/11336/33539

Matossian, B. (2015). División social del espacio residencial y migraciones. El caso de San Carlos de Bariloche. Revista EURE (Santiago), 41(124), 163-184. https://doi.org/10.4067/ s0250-71612015000400008

Medina, V. D. (2017). Las movilidades poblacionalesy su impacto territorial en la estructura espacial de las ciudades turísticas. El caso de San Carlos de Bariloche. EURE (Santiago), 43(129), 71-92. https://doi.org/10.4067/s0250-71612017000200004

Monasterio, H. (2006). Medición de la economía formal de San Carlos de Bariloche-Estimación del PBI 2005. Centro de Estudios Regionales (CER); Universidad FASTA.

Oglietti, G. y Colino, E. (29-31 de agosto de 2011). El ciclo de vida del destino turístico Bariloche. Particularidades del caso, limitaciones del enfoque y la contribución explicativa de la cepa turística de la enfermedad holandesa [Sesión de conferencia]. III Congreso anual de la Asociación de Economía para el Desarrollo de Argentina (AEDA). Buenos Aires, Argentina.

Ordenanza 2733 de 2016 [Concejo Municipal de San Carlos de Bariloche] Derecho de Participación en la Renta Diferencial Urbana. 23 de mayo de 2016.
Ordenanza 2871 de 2017 [Concejo Municipal de San Carlos de Bariloche]. Cambio de indicadores urbanísticos y nueva zonificación inmuebles 19-3A-41-01, 19-3A-4201A, 19-3A-42-01D y 19-3A-42-02A propiedad Las Victorias S.R.L, convenio transferencia de tierra por Derecho de Participación de la Renta Diferencial Urbana, emplazamiento de la nueva terminal de ómnibus. 26 de julio de 2017.

Ordenanza 2973 de 2018. [Concejo Municipal de San Carlos de Bariloche]. Obra Pública por contribución de mejoras, Fondo Municipal de Desarrollo Urbano. 19 de septiembre de 2018.

Páramo Lopera, C. y López-Morales, E. (2020). Principios, progresividad y factibilidades de la recuperación de "plusvalías" urbanas en el Chile actual. Revista de Geografía Norte Grande, (76), 121-142. http://dx.doi. org/10.4067/S0718-34022020000200121

Peinado, G., Barenboim, C. A., Nicastro, M. I. y Lagarrigue, P. A. (2018). Recuperación de plusvalías urbanas y sus impactos distributivos. Las compensaciones por mayor aprovechamiento urbanístico a raíz de convenios urbanísticos en la ciudad de Rosario, Argentina. Sociedady Economía, (35), 50-77. https://doi.org/10.25100/sye.v0i35.7295

Perló Cohen, M. y Zamorano Ruiz, L. (2001). Se justifica la aplicación del impuesto a la plusvalía en México. En M. Smolka y F. Furtado (Eds.), Recuperación de plusvalías en América Latina: Alternativas para el desarrollo urbano (pp. 149-172). EureLibros, Pontificia Universidad Católica de Chile; Lincoln Institute of Land Policy.

Pons Giner, B. (8-9 de junio de 2015). Colaboración públicoprivada y captación de plusvalías en el desarrollo de parques lineales en corredores fluviales urbanos: lecciones aprendidas de la regeneración del frente costero de Rosario (Argentina) [Sesión de conferencia]. VII Seminario Internacional de Investigación en Urbanismo. Departament d'Urbanisme i Ordenació del Territori. Universitat Politècnica de Catalunya, Barcelona-Montevideo, España-Uruguay. 
Reese, E. (2014). Construcción del territorio, mercado y política. La experiencia de la ley de hábitat popular en la Provincia de Buenos Aires. En D. Gargantini (Comp.), Hábitat, acceso al suelo y financiamiento. Experiencias alternativas de producción socio-habitacional (pp. 1317). Universidad Nacional de Córdoba

Sandroni, P. (2001). Plusvalías urbanas en Brasil: creación, recuperación y apropiación en la ciudad de São Paulo. En M. Smolka y F. Furtado (Eds.), Recuperación de plusvalías urbanas en América Latina. Alternativas para el desarrollo urbano (pp. 37-70). Eurelibros, Pontificia Universidad Católica de Chile; Lincoln Institute of Land Policy.

Smolka, M. O. (2013). Implementación de la recuperación de plusvalías en América Latina: políticas e instrumentos para el desarrollo urbano. https://www.lincolninst.edu/ sites/default/files/pubfiles/implementacion-recuperacionde-plusvalias-full_0.pdf

Soria Caballero, G. X. (2019). La plusvalía urbana como mecanismo para mejorar el financiamiento de infraestructura pública en la ciudad de Nuevo Chimbote, 2018 [Tesis de Maestría, Universidad de San Pedro]. Repositorio institucional. http://repositorio.usanpedro. edu.pe/handle/USANPEDRO/11007 